\title{
Tropical pulmonary eosinophilia: effect of addition of corticosteroids after failure of diethylcarbamazine therapy
}

The authors declare no financial disclosure

\begin{abstract}
Successful response in diethylcarbamazine (DEC) therapy in tropical pulmonary eosinophilia (TPE) is not universal with a $20-40 \%$ failure rates in chronic cases. Corticosteroids have been used in such patients. However, their role in management remains ill-defined. A patient of TPE with incomplete clinical, haematological and physiological response to a standard 3 weeks DEC therapy received additional corticosteroids for the next two cycles, after which complete remission occurred. However, there was a relapse two months later with evidence of a chronic state requiring further treatment with corticosteroids with good response.
\end{abstract}

Key words: tropical pulmonary eosinophilia, diethylcarbamazine, corticosteroids

Adv Respir Med 2017; 85: 51-54

\section{Introduction}

Tropical pulmonary eosinophilia (TPE) is an eosinophilic lung disease associated with a hypersensitivity response to microfilariae of the parasites, Wuchereria bancrofti and Brugia malayi [1]. It is fairly common in areas with filarial endemicity including regions of the Indian subcontinent, South East Asia, South America and Africa. It occurs mostly in the age group of 15-40 years with a male: female ratio of 4:1 [1].

The criteria for diagnosis of TPE include residence in an area endemic for filariasis, symptoms of recent onset of paroxysmal nocturnal cough with or without sputum, absolute blood eosinophil count of $2,000 / \mu$ l or above, absence of circulating microfilaria in blood, and successful clinical and haematological remission with diethylcarbamazine (DEC) therapy [1]. A standard treatment of DEC is $3-5 \mathrm{mg} / \mathrm{kg}$ body weight for 3 weeks.

However, $20-40 \%$ failure rates have been reported with DEC therapy [2]. Corticosteroids have been used in such patients with variable success. Though usually considered an acute and treatable disease, a chronic state has also been described in TPE [2]. We report a patient with treatment failure with DEC who responded to corticosteroids but the disease was found to evolve into a chronic state on serial follow-up. An evolution from acute to a chronic state has not been documented in TPE.

\section{Case report}

A 45-year-old non-smoker female presented with a two-year history of paroxysmal dry cough and exertional breathlessness without any wheeze, fever and constitutional symptoms. Clinical examination was not remarkable. Blood counts showed a total white cell count of $17700 / \mathrm{mm}^{3}$ with $32 \%$ eosinophils and an eosinophil count (AEC) of $5800 / \mathrm{mm}^{3}$. Serum total IgE was 6240 $\mathrm{IU} / \mathrm{ml}$. The chest radiograph (PA view) showed bilateral diffuse nodular shadows (Fig. 1). High resolution computed tomography (HRCT) scan

Address for correspondence: Sunil K. Chhabra, Department of Pulmonary Medicine, Vallabhbhai Patel Chest Institute, University of Delhi, India, e-mail: skchhabra@mailcity.com DOI: 10.5603/ARM.2017.0010

Received: 13.08.2016

Copyright (C) 2017 PTChP

ISSN 2451-4934 


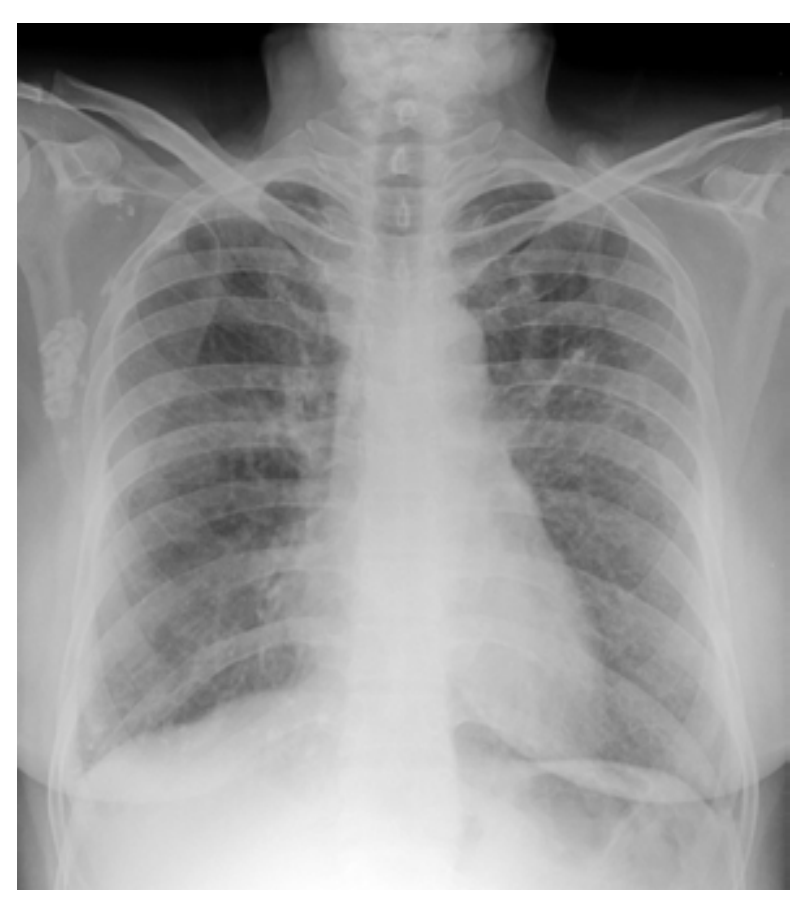

Figure 1. Chest radiograph (PA view) of the patient showing bilateral nodular shadows

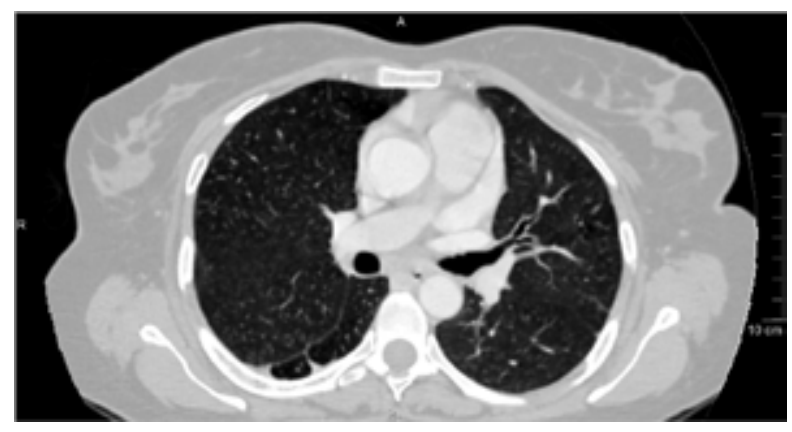

Figure 2. HRCT chest of the patient showing bilateral diffuse randomly distributed nodules

showed bilateral diffuse, randomly scattered micro-nodular opacities without any lobar predilection (Fig. 2). Smears and cultures of sputum obtained after induction were negative for pyogenes and acid fast bacilli. Blood test for micro-filarial antigen was negative but serology revealed positive titre of IgG anti-filarial antibodies. Commercial kits were used for serological assays. Spirometry showed a moderately severe restrictive pattern. Details of blood investigations are shown in Table 1.

Serum precipitins and immunoglobulins E and $\mathrm{G}$ (IgE and IgG) against Aspergillus species, and skin prick test for type 1 hypersensitivity to Aspergillus antigens were negative. Fibreoptic bronchoscopy was not remarkable and a transbronchial lung biopsy was non-contributory.

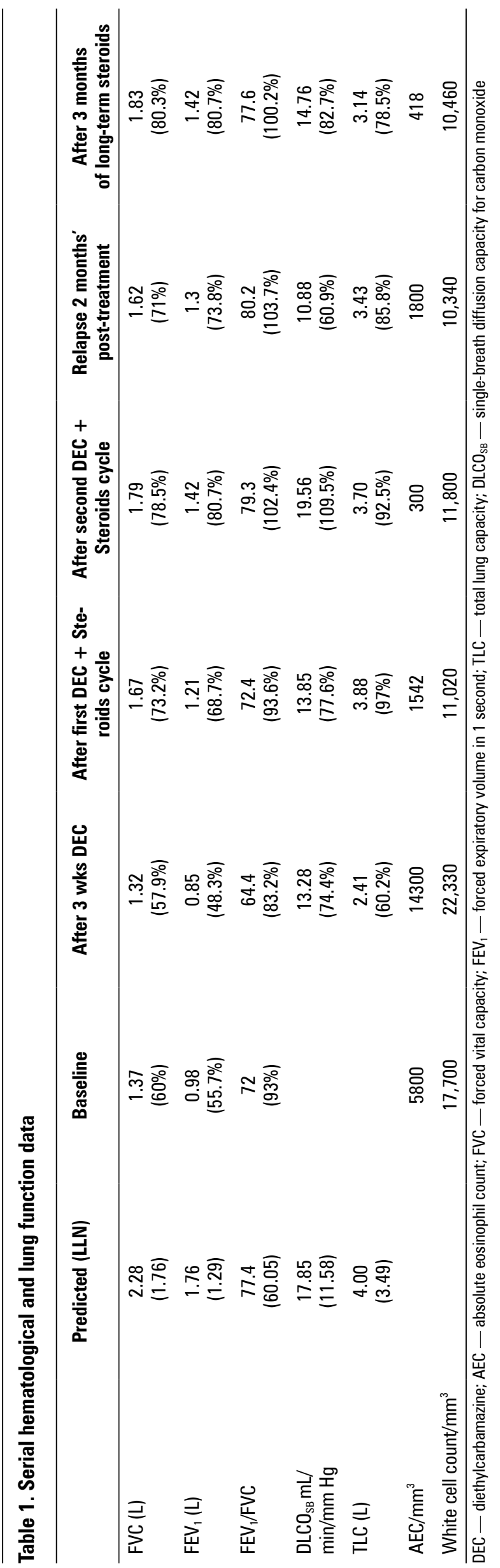


Bronchoalveolar lavage showed 81\% eosinophils. A diagnosis of TPE was made on the basis of the established criteria [1, 2]. DEC therapy was started, 100mg thrice daily, for three weeks.

There was only a partial relief in symptoms and the AEC was found to be increased (Table 1). The chest radiograph showed no remarkable change while there was a slight worsening on spirometry. Total lung capacity (TLC) estimation by closed circuit helium dilution confirmed restriction. Single breath diffusion capacity for carbon monoxide (DLCO) was in the normal range. A second cycle of DEC was initiated but this time with added corticosteroids (20 mg prednisolone). The symptoms diminished considerably after three weeks' treatment with a decline in peripheral eosinophilia. Substantial improvement in FVC and $\mathrm{FEV}_{1}$ occurred though these were still reduced but the TLC recovered to the normal range. A second cycle of DEC plus prednisolone in the same doses for 3 weeks resulted in symptomatic remission, disappearance of peripheral eosinophilia and normalization of lung function. The treatment was stopped.

The patient again developed exertional breathlessness two months later with increased AEC. Spirometry and TLC measurements showed mild restriction and this time, the DLCO was also moderately reduced. HRCT chest showed bilateral diffuse, randomly scattered micro-nodular opacities, though less profuse than at baseline. In view of the radiological abnormality and a deteriorating lung function picture with diffusion impairment, a diagnosis of chronic lung disease due to TPE was made and the patient was advised to take long term corticosteroids, starting with 30mg per day of oral prednisolone that was gradually tapered over the next 3 months. There was a substantial improvement in symptoms and AEC (Table 1). Serum anti-filarial antibodies became negative. Spirometry parameters and DLCO returned to the normal range but TLC remained below the normal limits, indicating persistent restrictive ventilator impairment.

\section{Discussion}

TPE occurs as a hypersensitivity reaction to the microfilariae of the parasites, Wuchereria bancrofti and Brugia malayi [1]. It predominantly affects the lungs. The respiratory symptoms include cough, breathlessness, wheezing and chest pain. Symptoms are usually more prominent at night. Systemic symptoms include fever, weight loss, fatigue and malaise. Wheezes and crackles may be found on examination although 20 per cent of patients may have no findings on examination [2]. Histopathologically, acute eosinophilic and histiocytic infiltration, eosinophilic bronchopneumonia and eosinophilic abscesses characterize the initial phases of the disease with generally well-marked fibrous tissue formation between six months to two years in more chronic cases [3]. Fibrosis is usually interstitial but also be peribronchial and perivascular in distribution. It is slowly progressive. Later in the natural course, the lung is badly scarred with a restrictive pattern though never as crippling as in idiopathic pulmonary fibrosis [2]. Patients who have frequent relapses or responded poorly to DEC are more likely to have impaired lung functions with progress to interstitial fibrosis. Spirometry is usually mixed restrictive-cum-obstructive with impaired diffusing capacity in chronic cases who may manifest mild hypoxemia due to ventilation -perfusion mismatch.

About 20 per cent of patients with TPE may have a normal chest radiograph. The main radiological features include reticulo-nodular shadows more in the mid-to-lower zones and miliary mottling resembling that seen in tuberculosis. HRCT scan often reveals miliary nodular shadows, bronchiectasis, air trapping and interstitial shadows, and, rarely, lymphadenopathy, cavitation, consolidation or pleural effusions. Though arsenic compounds were used earlier, short courses of two or three weeks of DEC have remained the cornerstone of therapy for the last five decades [2]. Chronic or relapsing cases may have persistent or incomplete clinical and radiological resolution and may require such courses frequently. Role of corticosteroids remains undefined [4].

An incomplete response to DEC has been reported earlier in TPE $[1,3,5,6]$ and may occur due to resistance to therapy, inadequate duration of therapy or a transition into a chronic inflammatory state. While resistance to DEC has not been documented, Vijayan et al. [6] provided evidence of a chronic inflammatory state on bronchoalveolar lavage in patients with treatment failure with DEC. A mild eosinophilic inflammation of the lower respiratory tract was found with cells spontaneously releasing increased amounts of superoxide anion and hydrogen peroxide. One-week treatment with prednisone significantly reduced the eosinophil inflammation and the release of oxidants [4]. In a recent review of natural history of TPE, Mullerpattan et al. [1] have also provided evidence of the development of a chronic lower airway and interstitial inflammatory state 
followed by diffuse interstitial fibrosis. In such chronic patients, DEC may be ineffective in up to $20-40 \%$ cases.

Corticosteroids have potent anti-inflammatory effects resulting from blocking of promoter sites of pro-inflammatory genes, recruiting of transcription factors to promoter sequences of genes coding for anti-inflammatory gene products and inhibition of the synthesis of almost all known cytokines [7, 8]. These drugs are also very effective in several eosinophilic lung diseases because of potent actions against the eosinophils, including promotion of eosinophil apoptosis and inhibition of degranulation of eosinophils $[9,10]$. This explains their efficacy in TPE. It is also possible that the chronic interstitial inflammatory state represents a toxic response to DEC that responds to corticosteroids.

Antifilarial antibodies were not monitored during treatment except at the beginning and at the end of the treatment. The response in TPE is usually ascertained by symptoms and resolution of peripheral eosinophilia. In the present case, incomplete radiological resolution, relapse of peripheral eosinophilia and persistent restrictive abnormality of lung function after continued corticosteroid therapy suggested a transition into a chronic state. Thus, TPE may not be viewed as a benign and curable acute condition but one with a potential to progress to a chronic lung disease. This provides the rationale for corticosteroid therapy, especially in relapsing or chronic cases of TPE [2, 4]. The dose and duration of therapy in the long term management of TPE needs to be established.

\section{Conflict of interest}

The authors declare no conflict of interest.

\section{References}

1. Mullerpattan JB, Udwadia ZF, Udwadia FE. Tropical pulmonary eosinophilia--a review. Indian J Med Res. 2013; 138(3) 295-302, doi: 10.1016/s0954-6111(05)80308-7, indexed in Pubmed: 24135173.

2. Vijayan VK. Immunopathogenesis and treatment of eosinophilic lung diseases in the tropics. In: Sharma OP (ed). Lung biology in health and disease: tropical lung disease, $2^{\text {nd }}$ ed. New York: Taylor and Francis 2006: 195-239.

3. Rom WN, Vijayan VK, Cornelius MJ, et al. Persistent lower respiratory tract inflammation associated with interstitial lung disease in patients with tropical pulmonary eosinophilia following conventional treatment with diethylcarbamazine. Am Rev Respir Dis. 1990; 142(5): 1088-1092, doi: 10.1164/ ajrccm/142.5.1088, indexed in Pubmed: 2173455.

4. Vijayan VK. Role of steroids in „chronic” tropical eosinophilia-A bronchoalveolar lavage study. Indian J Chest Dis Allied Sci. 1987; 7: 29.

5. Vijayan VK, Rao KV, Sankaran K, et al. Tropical eosinophilia: clinical and physiological response to diethylcarbamazine. Respir Med. 1991; 85(1): 17-20, doi: 10.1016/s09546111(06)80205-2, indexed in Pubmed: 1901660.

6. Vijayan VK, Sankaran K, Venkatesan P, et al. Effect of diethylcarbamazine on the alveolitis of tropical eosinophilia. Respiration. 1991; 58(5-6): 255-259, doi: 10.1159/000195941, indexed in Pubmed: 1792413.

7. Scheinman RI, Cogswell PC, Lofquist AK, et al. Role of transcriptional activation of I kappa B alpha in mediation of immunosuppression by glucocorticoids. Science. 1995; 270(5234): 283-286, doi: 10.1126/science.270.5234.283, indexed in Pubmed: 7569975 .

8. Almawi WY, Beyhum HN, Rahme AA, et al. Regulation of cytokine and cytokine receptor expression by glucocorticoids. J Leukoc Biol. 1996; 60(5): 563-572, indexed in Pubmed: 8929546.

9. Meagher LC, Cousin JM, Seckl JR, et al. Opposing effects of glucocorticoids on the rate of apoptosis in neutrophilic and eosinophilic granulocytes. J Immunol. 1996; 156(11): 44224428, indexed in Pubmed: $\underline{8666816}$.

10. Kita H, Abu-Ghazaleh R, Sanderson CJ, et al. Effect of steroids on immunoglobulin-induced eosinophil degranulation. J Allergy Clin Immunol. 1991; 87(1 Pt 1): 70-77, doi: 10.1016/00916749(91)90214-9, indexed in Pubmed: 1991924. 